OPEN ACCESS

Edited by:

Vinicius Maracaja-Coutinho,

Universidad de Chile, Chile

Reviewed by:

Daniel Goldman,

University of Western Ontario, Canada

Steve McKeever,

Uppsala University, Sweden

${ }^{*}$ Correspondence:

Vinod Suresh

v.suresh@auckland.ac.nz

Specialty section:

This article was submitted to Computational Physiology and

Medicine,

a section of the journal

Frontiers in Physiology

Received: 14 December 2018 Accepted: 19 March 2019

Published: 12 April 2019

Citation:

Afshar N, Safaei S, Nickerson DP,

Hunter PJ and Suresh V (2019)

Computational Modeling of Glucose

Uptake in the Enterocyte.

Front. Physiol. 10:380.

doi: 10.3389/fphys.2019.00380

\section{Computational Modeling of Glucose Uptake in the Enterocyte}

\author{
Nima Afshar ${ }^{1}$, Soroush Safaei ${ }^{1}$, David P. Nickerson ${ }^{1}$, Peter J. Hunter ${ }^{1}$ and Vinod Suresh ${ }^{1,2 *}$ \\ ${ }^{1}$ Auckland Bioengineering Institute, University of Auckland, Auckland, New Zealand, ${ }^{2}$ Department of Engineering Science, \\ University of Auckland, Auckland, New Zealand
}

Absorption of glucose across the epithelial cells of the small intestine is a key process in human nutrition and initiates signaling cascades that regulate metabolic homeostasis. Validated and predictive mathematical models of glucose transport in intestinal epithelial cells are essential for interpreting experimental data, generating hypotheses, and understanding the contributions of and interactions between transport pathways. Here we report on the development of such a model that, in contrast to existing models, incorporates mechanistic descriptions of all relevant transport proteins and is implemented in the CellML framework. The model is validated against experimental and simulation data from the literature. It is then used to elucidate the relative contributions of the sodium-glucose cotransporter (SGLT1) and the glucose transporter type 2 (GLUT2) proteins in published measurements of glucose absorption from human intestinal epithelial cell lines. The model predicts that the contribution of SGLT1 dominates at low extracellular glucose concentrations $(<20 \mathrm{mM})$ and short exposure times $(<60 \mathrm{~s})$ while the GLUT2 contribution is more significant at high glucose concentrations and long durations. Implementation in CellML permitted a modular structure in which the model was composed by reusing existing models of the individual transporters. The final structure also permits transparent changes of the model components and parameter values in order to facilitate model reuse, extension, and customization (for example, to simplify, or add complexity to specific transporter/pathway models, or reuse the model as a component of a larger framework) and carry out parameter sensitivity studies.

Keywords: computational modeling, glucose uptake, SGLT1, GLUT2, CelIML, OpenCOR

\section{INTRODUCTION}

Almost all of the nutrients, electrolytes, and water from food are absorbed into blood capillaries through the mucosa of the small intestine. Most absorption processes in the small intestine are driven by an electrochemical gradient of ions across the boundary of epithelial cells (enterolyses) lining the lumen. Transporter proteins embedded in the apical membrane carry ions and nutrients into the enterocyte. Other transporters in the basolateral membrane then extrude the ions into the interstitial space from where they enter capillary blood by diffusion. Carbohydrates are the main source of energy in the body. They break down to monosaccharides like glucose, which is the most important carbohydrate fuel in the cell. Therefore the uptake and transport of glucose through the small intestine epithelial cells is a vital aspect of human nutrition. Subsequent transport and metabolism of the absorbed species triggers responses such as hormone release, appetite regulation and growth via complex physiological feedback pathways. A mechanistic understanding 
of these pathways and how they are disrupted in disease is lacking, partly due to the difficulties of making experimental measurements in the luminal and capillary compartments. A validated computational model of the absorption pathways can overcome these difficulties by providing quantitative predictions of concentrations and transport rates in the lumen and cell compartments (Hunter and Borg, 2003; Ingalls, 2012).

Many studies in the past few decades have focussed on mathematical modeling of the glucose-insulin control system in order to study how metabolism and the regulatory system are disrupted in diseases like diabetes (reviewed in Palumbo et al., 2013). At the cellular level, models of glucose uptake and transport in the kidneys (Weinstein, 2015), glucose homeostasis in the liver (König et al., 2012), and glucose sensing (Riz and Pedersen, 2015) have been developed. In contrast, mathematical modeling of glucose uptake by the enterolyses lining the small intestinal mucosa has attracted little attention. The first model of glucose transport in the enterocyte was developed by Thorsen et al. (2014). The model focussed on the regulation of $\mathrm{Na}, \mathrm{K}-$ ATPase in enterolyses during glucose absorption. It considered SGLT1 as the sole pathway for glucose entry into the cell at the apical membrane and studied how the intracellular $\mathrm{Na}+$ concentration can be maintained in the face of SGLT1-associated $\mathrm{Na}+$ influx. One limitation of the model is the absence of a GLUT2 pathway for glucose entry at the apical membrane. The role of apical GLUT2 is still a matter of controversy with some studies indicating its presence and importance for glucose uptake (Kellett and Brot-Laroche, 2005; Zheng et al., 2012) while others have suggested SGLT1 as the dominant or sole pathway (Gorboulev et al., 2012; Röder et al., 2014). Differences in experimental conditions and data interpretation are partly the reason for lack of consensus (Kellett, 2012; Koepsell and Gorboulev, 2012). In this work, we developed a mathematical model that includes apical GLUT2 and parameterized it against published experimental data. We then used the model to examine the relative contributions of SGLT1 and GLUT2 in published cell culture data on glucose uptake (Zheng et al., 2012). Finally we assessed the impact of increased glucose transporter expression on uptake rates in diabetes.

The Thorsen model incorporated a mixture of mechanistic transporter models (e.g., SGLT1, basolateral GLUT2), empirical flux expressions (e.g., NaK-ATPase, an effective $\mathrm{Na}-\mathrm{Cl}$ cotransporter), and diffusive membrane fluxes for $\mathrm{Na}+\mathrm{K}+$, and $\mathrm{Cl}$. We modified this framework to explicitly incorporate mechanistic models of all relevant transporters. In particular, we replaced the $\mathrm{Na}-\mathrm{Cl}$ co-transporter in the original model with individual models for the anion exchanger 1 (AE1) and $\mathrm{Na}+$ $/ \mathrm{H}+$ exchanger (NHE3) proteins at the apical membrane and incorporated ENaC and CFTR channels for apical $\mathrm{Na}+$ and $\mathrm{Cl}-$ transport. This makes it possible to use the model to study scenarios where the expression and/or function of these transport proteins is altered, for example in gene knockout/mutation studies or the use of channel inhibitors and agonists.

The model is implemented in the open source, extensible markup language (XML)-based CellML modeling environment used to represent mathematical models of biology based on ordinary differential and algebraic equations (Cuellar et al.,
2003). We adopted a modular, compositional approach to model construction by reusing CellML models of individual transport proteins encoded in an online, curated repository [Physiome Model Repository (PMR, models.physiomeproject. org)] to facilitate the sharing of models ( $Y u$ et al., 2011). The complete model, including parameter values, simulation software and simulation conditions, can be downloaded from PMR with the following link: https://models.physiomeproject. org/workspace/572.

\section{METHODS}

\subsection{Model Construction}

We constructed a mathematical model of the epithelial cell of a small intestine (enterocyte) that incorporates the relevant transport proteins identified in the literature (Barrett and Keely, 2015) and diffusion pathways (Figure 1). The membrane localization and function of these transporters and the source of the original mathematical models are listed in Table 1. The apical (luminal) and basolateral (interstitial) surface of the cell are in contact with distinct extracellular compartments. Transport of substances occurs across the membranes as well as directly between the extracellular compartments across the paracellular junctions. The variables to be solved in the model are chemical species $\left(\mathrm{Na}^{+}, \mathrm{K}^{+}, \mathrm{H}^{+}, \mathrm{Cl}^{-}, \mathrm{HCO}_{3}^{-}\right.$, glucose) concentrations in each compartment and the two membrane potentials. Flux balance and electric charge conservation laws yield the governing equations of the model. Water transport is not included and hence we limit ourselves to modeling iso-osmotic transport. Model equations are provided in the Supplementary Material.

The model was implemented in the open-source, modular CellML framework. CellML is an XML based language commonly used to encode and simulate mathematical models based on algebraic and ordinary differential equations. Encoded models are available in an online, curated repository [Physiome Model Repository-PMR(models.physiomeproject.org)] (Yu et al., 2011). Reuse of models and components within models is possible through the use of the import element that enables encapsulation of other CellML files within a CellML model and facilitates a modular, compositional approach to the construction of complex models. The application of this approach in the enterocyte model is shown in Figure 2. Existing models of the individual transporters were imported into the top level model file (modular_model.cellml). Units and parameters for all components as well as initial conditions for specific simulations were specified in separate . cellml files and also imported into the top level file. The models were encapsulated as a group into the enterocyte component in which the overall balance equations for the chemical species and electric currents were coded. The mappings element links variables that are common between the different components, e.g., glucose concentrations in GLUT2.cellml, SGLT1.cellml and enterocyte. The environment component comprises independent variables that are common to all components, which in this case, is solely time.

The model was coded and simulated in OpenCOR (Version 0.5) (Garny and Hunter, 2015). The CVODES solver was used 


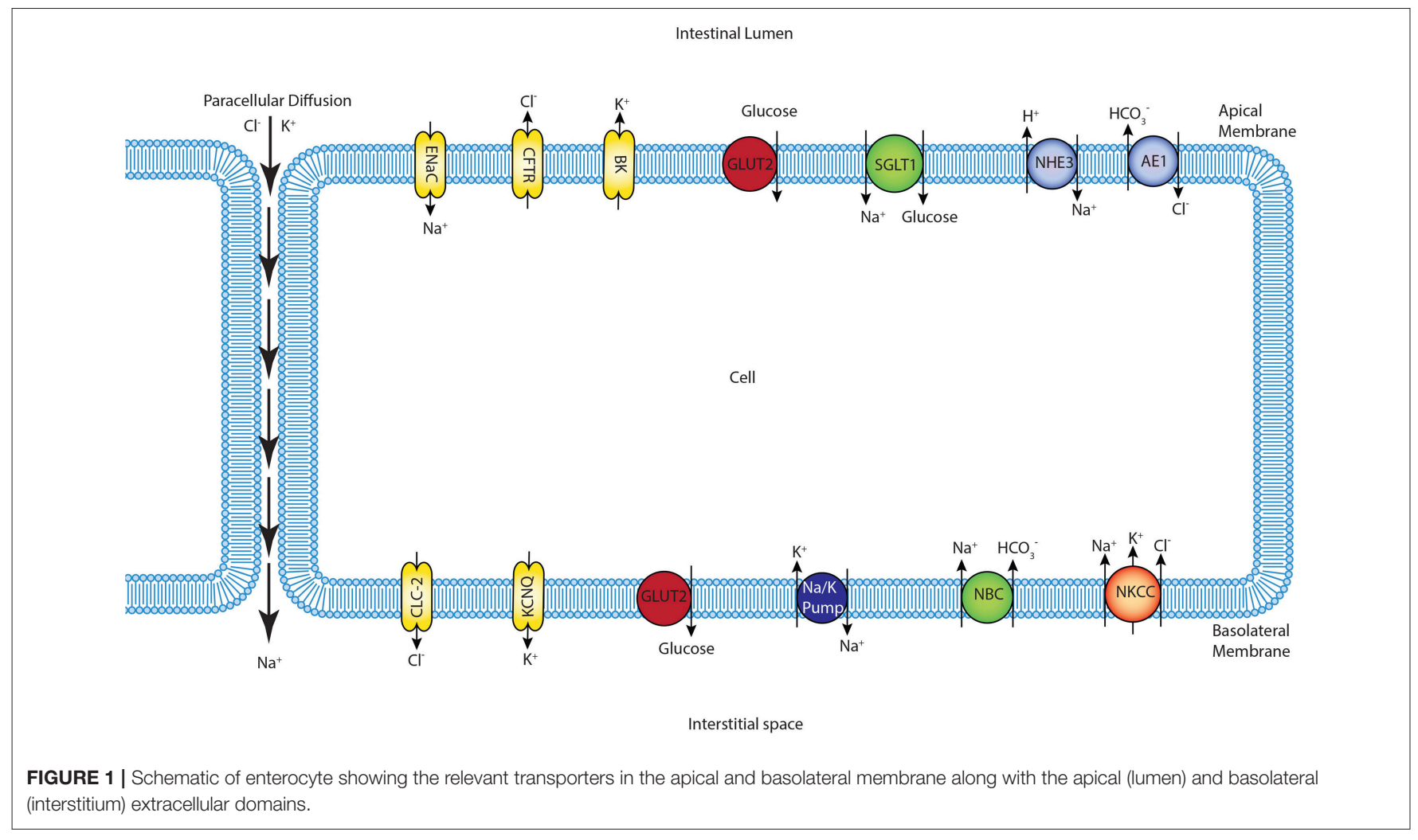

TABLE 1 | List of transporters used in the model along with their locations and roles.

\begin{tabular}{|c|c|c|c|c|}
\hline Transporter & Location & Role & Chemical Species & $\begin{array}{l}\text { Source of the mathematical } \\
\text { model }\end{array}$ \\
\hline SGLT1 & Apical & Cotransporter & 1 Glucose, $2 \mathrm{Na}^{+}$ & Parent et al., 1992 \\
\hline GLUT2 & Apical and Basolateral & Uniporter Protein & Glucose & Pradhan et al., 2013 \\
\hline NHE3 & Apical & Antiporter & $1 \mathrm{Na}^{+}, 1 \mathrm{H}^{+}$ & Weinstein, 1995 \\
\hline CFTR & Apical & Channel & $\mathrm{Cl}^{-}$ & Fong et al., 2016 \\
\hline CLC-2 & Basolateral & Channel & $\mathrm{Cl}^{-}$ & Fong et al., 2016 \\
\hline $\mathrm{ENaC}$ & Apical & Channel & $\mathrm{Na}^{+}$ & Fong et al., 2016 \\
\hline $\mathbb{K}$ & Basolateral & Channel & $\mathrm{K}^{+}$ & Fong et al., 2016 \\
\hline NBC & Basolateral & Cotransporter & $1 \mathrm{Na}^{+}, 3 \mathrm{HCO}_{3}^{-}$ & Østby et al., 2009 \\
\hline
\end{tabular}

with the BDF integration method and Newton iterations. All of the models including their parameters can be downloaded from PMR with the following link: https://models.physiomeproject. org/workspace/572.

\subsection{Comparison With Experiments}

The model was validated against published experimental measurements of glucose uptake in the human enterocytelike cell lines Caco-2 and IEC6 Zheng et al. (2012). In the experiments, the cells were cultured on impermeable surfaces for 10-15 days in high glucose $(25 \mathrm{mM})$ medium. To measure glucose uptake, varying concentrations $(0.5-50 \mathrm{mM})$ of glucose were introduced into the apical chamber in a buffer solution with a baseline composition $130 \mathrm{mM} \mathrm{NaCl}, 4 \mathrm{mM} \mathrm{KH}_{2} \mathrm{PO}_{4}, 1$ $\mathrm{mM} \mathrm{CaCl}_{2}$. The osmolarity of the buffer was maintained during the measurements by modulating the $\mathrm{NaCl}$ content such that if the glucose concentration was $x \mathrm{mM}, \mathrm{NaCl}$ concentration was $130-x / 2 \mathrm{mM}$. After exposure to the glucose stimulus for different durations (30-600 s), cells were lysed and intracellular glucose and protein concentrations were measured. Since the measurements were reported in nanomole glucose per milligram (mg) protein, the data were converted to concentration units 


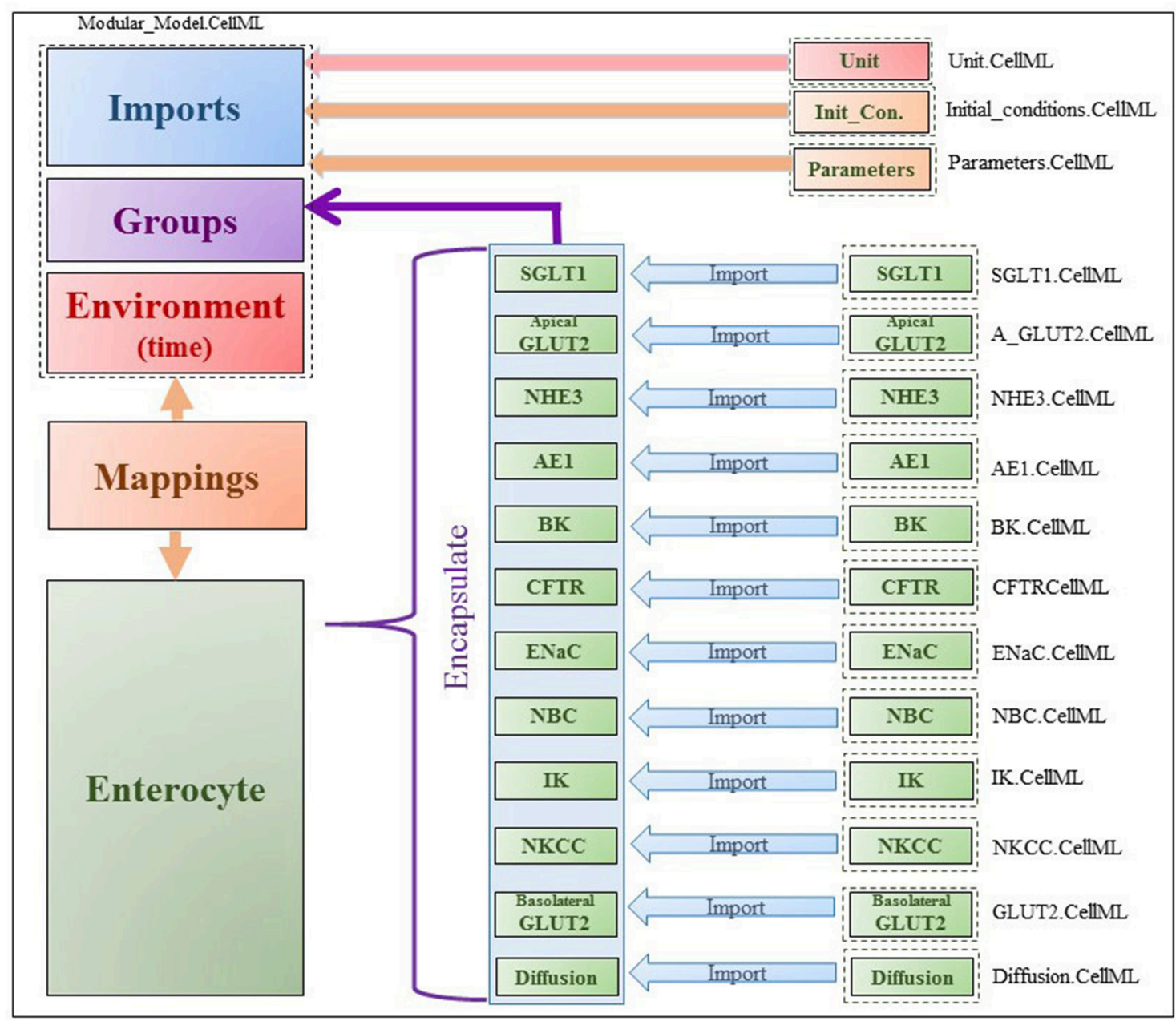

FIGURE 2 | Figure shows the modularity of CellML model. Encapsulation hierarchy (purple), the CellML model imports (blue) and the other key parts (units, parameters,components and mappings) of the top level CellML model.

(millimole per liter, $\mathrm{mM}$ ) by doing the unit conversion from nanomole $/ \mathrm{m}^{3}$ to $\mathrm{mM}$ and also multiplying by the cellular protein concentration ( $\mathrm{mg}$ protein per $\mathrm{ml}$ cell volume). The conversion factor $a$ (protein density) was used as a fitting parameter in a non-linear Generalized Reduced Gradient optimization to match model outputs to the data. The optimization was done using the Microsoft Excel Solver (Microsoft Office 2013) by minimizing the least square error between model predicted and measured intracellular glucose concentration.

In the simulations, the apical compartment was treated as an infinite bath of constant composition based on the experimental conditions. Since the cells were cultured on an impermeable substrate, the volume of the basolateral compartment $\left(V_{b}\right)$ was not measured. In the simulations, $V_{b}$ was fixed at different multiples $(m=0.1,1,10)$ of the cell volume $\left(V_{c}\right)$ and also as an infinite bath to generate a range of predictions. This allowed us to account for the uncertainty in the actual volume of the basolateral compartment. For finite values of $V_{b}$, the composition of the basolateral compartment cannot be regarded as constant and was instead determined by the flux of glucose/ions across the basolateral membrane. Since the experiments were conducted under iso-osmotic conditions, there is no water transfer between the compartments and hence $V_{b}$ and $V_{c}$ were held fixed for the duration of each simulation.

\section{RESULTS}

\subsection{Steady State and Dynamic Responses}

The model was first checked for physiological consistency by determining intracellular concentrations and membrane potentials in the absence and presence of a glucose stimulus. Steady state values of the model variables were computed with no glucose in the extracellular compartments. In these simulations, the composition of the apical and basolateral compartments were identical and held constant $\left(140 \mathrm{mM} \mathrm{Na}^{+}, 5.4 \mathrm{mM} \mathrm{K}^{+}, 103 \mathrm{mM}\right.$ $\mathrm{Cl}^{-}$). Results were consistent with reported values (Table 2).

Next, the dynamic response to an apical glucose stimulus was determined. The model was initialized in the steady state 
TABLE 2 | Reported values for intracellular ions concentration from simulated model and literature.

\begin{tabular}{|c|c|c|c|}
\hline Ion & Model result & $\begin{array}{l}\text { Reported } \\
\text { value }\end{array}$ & Reference \\
\hline $\mathrm{Na}^{+}(\mathrm{mM})$ & 61 & $45-65$ & $\begin{array}{l}\text { Nellans and Schultz, } \\
\text { 1976; Okada et al., } 1976\end{array}$ \\
\hline $\mathrm{K}^{+}(\mathrm{mM})$ & 127 & $120-40$ & $\begin{array}{l}\text { Okada et al., 1976; } \\
\text { Vogalis, } 2000\end{array}$ \\
\hline $\mathrm{Cl}^{-}(\mathrm{mM})$ & 69 & $50-70$ & $\begin{array}{l}\text { Frizzell et al., 1973; } \\
\text { Nellans et al., 1973; } \\
\text { Okada et al., } 1976\end{array}$ \\
\hline $\begin{array}{l}\text { Apical (lumen-cell) } \\
\text { membrane potential (mV) }\end{array}$ & -30 & $-36 \pm 0.5$ & Rose and Schultz, 1971 \\
\hline $\begin{array}{l}\text { Basolateral } \\
\text { (interstitium-cell) } \\
\text { membrane potential (mV) }\end{array}$ & -36 & $-40.5 \pm 0.8$ & Rose and Schultz, 1971 \\
\hline $\mathrm{pH}$ & 7.16 & 7.2 & Shimada and Hoshi, 1987 \\
\hline
\end{tabular}

described in Table 2 and a time dependent, extracellular glucose stimulus previously used in the literature (Thorsen et al., 2014) was applied at $t=60 \mathrm{~s}$ (Figure 3A). Other extracellular variables were maintained at the same values used for the previous set of simulations. The stimulus causes a depolarization of both membranes (Figure 3B). Membrane potentials recover rapidly to baseline after around $100 \mathrm{~s}$ and mirror the time course of the stimulus. Transient changes in the transepithelial potential difference $(\approx 1.4 \mathrm{mV}$ increase) are of the same direction and comparable magnitude to values reported in the literature (1.9 $\pm 0.1 \mathrm{mV}$;Rose and Schultz, 1971) while changes in the apical potential $(\approx 12 \mathrm{mV}$ increase) are higher than values reported in the same study $(6 \pm 0.5 \mathrm{mV})$ (Figures 3B,C). Intracellular ion concentrations and $\mathrm{pH}$ all exhibit a slower transient response than the membrane potentials that lasts for $\approx 200-300 \mathrm{~s}$.

\subsection{Comparison With the Thorsen et al. (2014) Model}

Since our model is similar to that developed by Thorsen et al. (2014), we compared the responses of both models when the same parameters (Table 3), initial conditions and glucose stimulus were used. Model outputs were normalized against the steady state values of the Thorsen model and are shown in Figure 4. A few observations may be made: in the absence of a glucose stimulus, steady state values of the membrane potentials in our model are around 30\% lower while the transepithelial potential is around $80 \%$ higher. Steady state values for concentration of chloride, potassium, and glucose are 5-10\% lower than the values in the previous model whereas for sodium it is about $10 \%$ higher. In response to a glucose stimulus, our model has a larger change in membrane potentials and intracellular glucose, but smaller changes in sodium and potassium. Chloride responses are of almost the same magnitude in both models. The duration of the transients are similar in both models, except for glucose where our model has a similar rise time, but a slower decay (around 2 times slower).

\subsection{Comparison Against Cell Culture Data}

Finally, model predictions were compared against measurements carried out in cell culture studies (Zheng et al., 2012). The experiments used Caco-2 and IEC6 cell lines. While Caco-2 expresses both SGLT1 and GLUT2, IEC6 cells do not express GLUT2. We therefore turned off the expression of GLUT2 in the apical membranes to simulate these cells.

Model predictions of the intracellular glucose concentrations are in good agreement with the measurements over the entire range of time points and apical glucose concentrations for both cell lines (Figure 5). As shown in Figures 5A,B at 30 and 60 $s$ of exposure, glucose concentrations in both cell lines have a tendency to level off at higher concentration of glucose in the apical compartment. IEC6 still has the same behavior for longer exposure times (300 and $600 \mathrm{~s}$ ) whereas concentrations in $\mathrm{Caco} 2$ do not saturate with increasing glucose concentration in the apical compartment (Figures 5C,D). The protein density parameter $a$ are quite close to each other for Caco-2 cell line and varies for IEC- 6 cell line to fit the four exposure durations (Table 4).

Together, these results indicate that the model is able to reproduce a range of independent experimental observations. Next we present applications of the validated model to address questions about glucose uptake pathways in health and disease.

\subsection{Role of Apical GLUT2 in Glucose Uptake}

In the original study of Zheng et al., the experimental data in Figures 5A-D were interpreted as indicating the presence of GLUT2-mediated uptake at the apical membrane (Zheng et al., 2012). We investigated if an alternative explanation was possible whereby SGLT1 expression levels in the model could be tuned to reproduce the same trends in intracellular glucose concentration. In Figures 6A-D, the data for Caco- 2 cells at the $600 \mathrm{~s}$ time point are compared to the model with varying levels of apical GLUT2 and SGLT1. The baseline model with normal expression of SGLT1 and apical GLUT2 provides a good fit to the data over the full range of apical glucose concentrations (Figure 6A). When apical GLUT2 is turned off with no changes in SGLT1 expression (Figure 6B), model predictions of intracellular glucose are low compared to the data for apical glucose concentrations higher than $10 \mathrm{mM}$. In addition, model predictions saturate after around $20 \mathrm{mM}$ of apical glucose while the data shows an increasing trend. A higher expression of SGLT1 was also examined and can provide a better match to the data in the absence of apical GLUT2. With no apical GLUT2 and 2-fold levels of baseline SGLT1 (Figure 6C) the model overpredicts the data at low apical glucose concentrations $(<10 \mathrm{mM})$ and underpredicts the data at apical glucose concentrations $>40 \mathrm{mM}$. When SGLT1 levels are increased to 3 times the baseline value, the model overpredicts the data over the whole range, except at a apical glucose of 50 $\mathrm{mM}$ (Figure 6D).

In order to explain these results, the contribution of SGLT1 and GLUT2 to the apical glucose flux is shown in Figure 7 following $600 s$ of exposure to apical glucose. It is seen that 

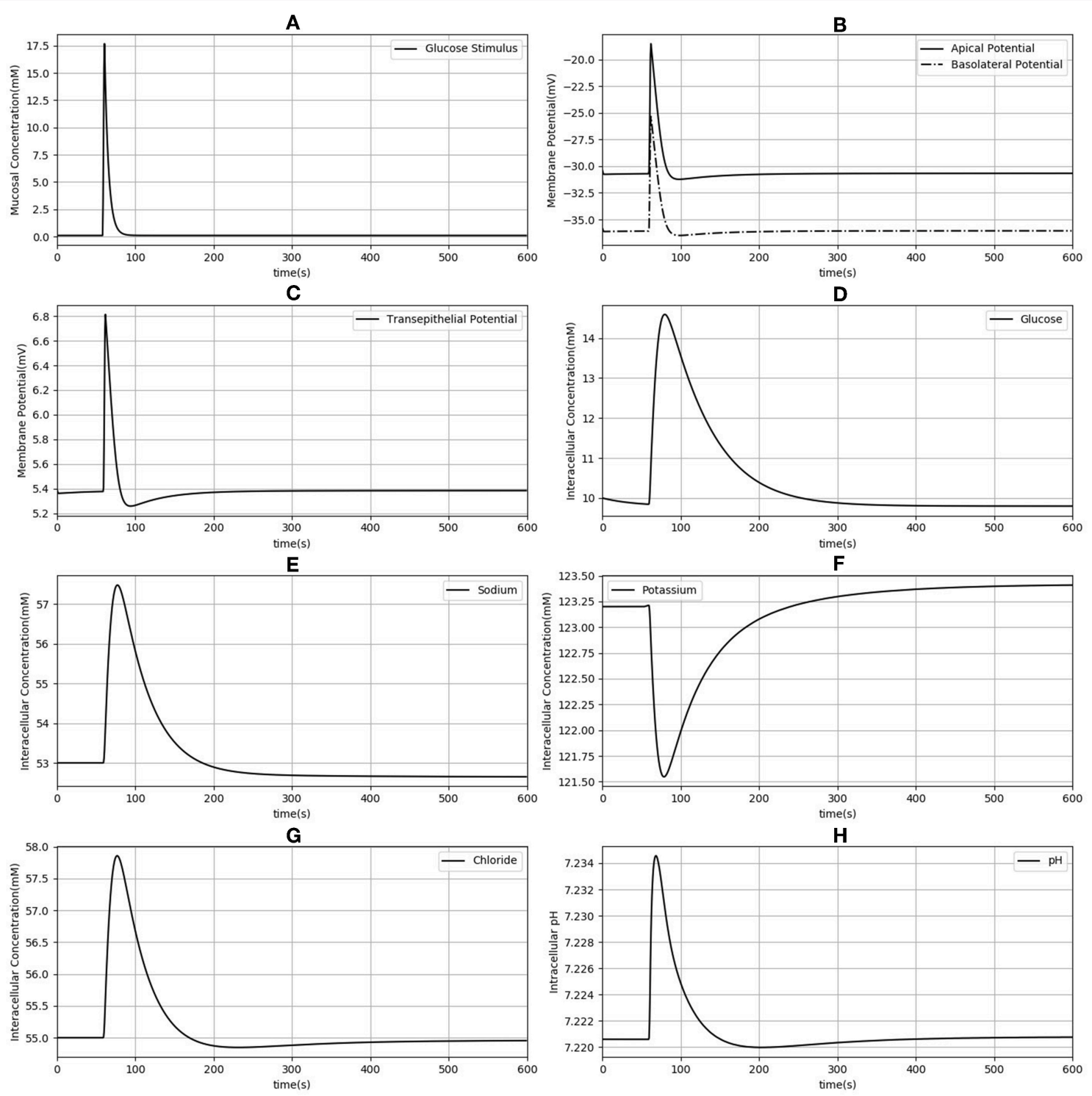

FIGURE 3 | Dynamic response of the model to an extracellular glucose stimulus. The stimulus consists of a step increase followed by an exponential decay (A). Apical and basolateral membrane potentials (B), transepithelial potential (C), and intracellular concentrations of glucose (D), sodium (E), potassium (F), chloride (G), and pH (H) are shown.

TABLE 3 | Parameter values used in the simulations.

\begin{tabular}{lccc}
\hline Parameter & $\begin{array}{c}\text { Value in our model } \\
\text { (Figures 3, 4) }\end{array}$ & $\begin{array}{c}\text { Value in our model } \\
\text { (Other figures) }\end{array}$ & Unit \\
\hline $\mathrm{nSGLT1}$ & $18 \times 10^{7}$ & $4 \times 10^{7}$ & - \\
$\mathrm{nA} \mathrm{GLUT2}_{\mathrm{n}}$ & 0 & $42 \times 10^{7}$ & - \\
$\mathrm{nB}_{\mathrm{GLUT2}}$ & $14 \times 10^{6}$ & $14 \times 10^{7}$ & - \\
$\mathrm{V}_{\text {cell }}$ & $6 \times 10^{-16}$ & $2 \times 10^{-15}$ & $\mathrm{~m}^{3}$ \\
Capacitance & $1 \times 10^{-5}$ & $1 \times 10^{-5}$ & $\mu \mathrm{F}$
\end{tabular}

for apical glucose concentrations up to around $25 \mathrm{mM}$, the flux through SGLT1 is higher than GLUT2 flux but after that it starts to saturate, while the GLUT2 flux continues to increase and get higher than SGLT1 flux. This behavior looks similar to the previous experimental study Kellett and Helliwell (2000) which at the apical glucose concentration of $50 \mathrm{mM}$ the glucose flux through GLUT2 is about 2 times higher than flux via SGLT1, Thus, varying the level of SGLT1 in the absence of apical GLUT2 is unable to capture the shape and magnitude of the experimental measurements since transport through SGLT1 saturates at an apical glucose concentration of about $25 \mathrm{mM}$. This suggests that apical GLUT2 is essential to account for the data from Zheng et al. (2012).

\subsection{Glucose Uptake in Diabetes}

In diabetes, expression levels of SGLT1 and GLUT2 in the small intestine are reported to be increased 3 to 4 -fold compared 

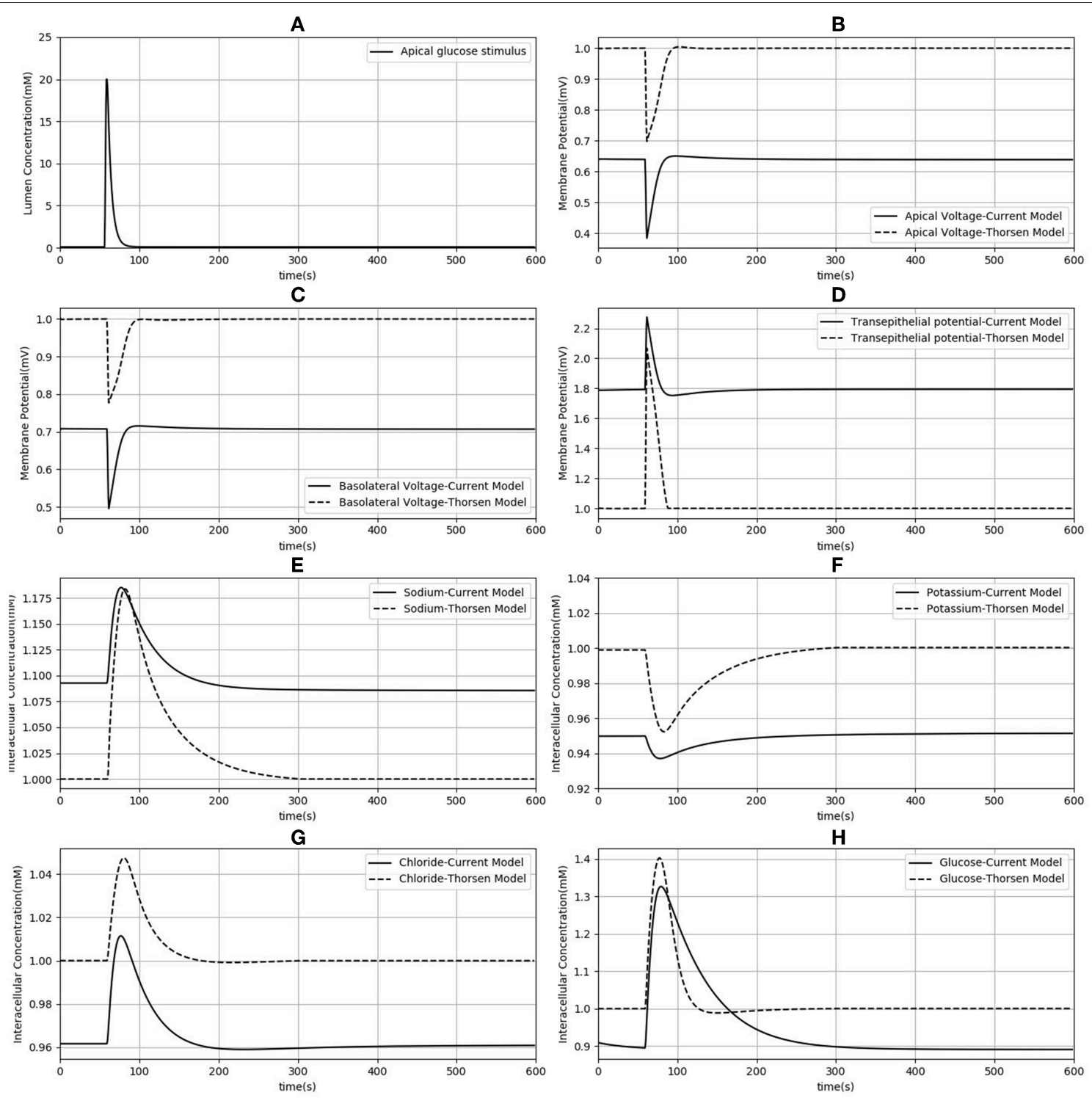

FIGURE 4 | Comparison of model responses against the model of Thorsen et al. (2014). Each variable has been normalized against the corresponding steady value from the Thorsen model. (A) Apical glucose stimulus. (B,C) Apical and basolateral membrane potential respectively. (D) Transepithelial potential. (E-H) Sodium, potassium, chloride and glucose intracellular concentration.

to non-diabetic controls in both human and animal studies (Fedorak et al., 1991; Burant et al., 1994; Dyer et al., 1997, 2002). The surface area of the villi has also been reported to increase in diabetes (Schedl and Wilson, 1971). Together these factors are expected to lead to higher rates of glucose absorption to the blood. However, the magnitude of the effect is not known. We used our developed model to study the effect of a 3-fold elevated SGLT1 and GLUT2 expression levels on glucose flux into the basolateral compartment. Figure 8 shows the ratio of steady state glucose flux into the basolateral compartment, normalized to the flux at baseline conditions over a range of apical glucose concentrations. The increase in glucose absorption is less than the increase in transporter expression levels. For apical glucose concentrations up to $50 \mathrm{mM}, 3$-fold increase in SGLT1 levels causes a small increase in the basolateral flux over the whole range of apical glucose concentration. This increase is $<1.1$ times the baseline value. On the other hand increasing the level of GLUT2 by 3 -fold increases the basolateral flux to almost 3 times the baseline value. This increase is observed over the whole range of glucose concentration. The result shows that higher levels of GLUT2 in diabetics may lead to a proportional increase in glucose absorption. In contrast, increases in SGLT1 cause a much smaller increase in absorption. However, SGLT1 may indirectly increase absorption rates since studies have shown that apical GLUT2 expression is dependent on SGLT1 activity (Kellett and Helliwell, 2000). 

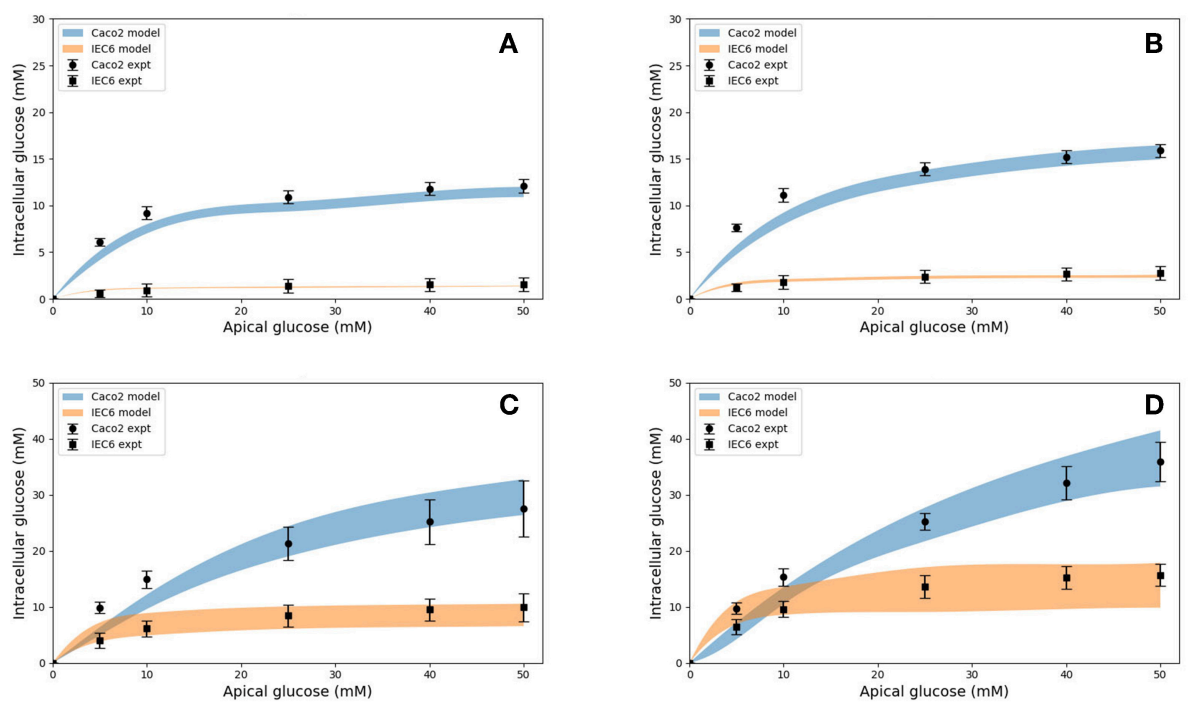

FIGURE 5 | Intracellular glucose concentrations for a range of extracellular glucose concentrations in Caco2 and IEC6 cells and exposure times (A: 30 s, B: 60 s, C: 300 s, D: 600 s). Experimental data points and error bars were digitally extracted from Zheng et al. (2012). Strips for the model predictions represent the range of values generated by setting $V_{b}=m V_{c}, m=0.1,1,10,100, \infty$.

TABLE 4 | Best fit values of the protein density (a) used to generate the simulated curves in Figure $\mathbf{5}$ for different exposure times and both cell lines.

\begin{tabular}{lcc}
\hline $\begin{array}{l}\text { Exposure duration } \\
\text { (seconds) }\end{array}$ & $\begin{array}{c}\boldsymbol{a} \text { (g protein/ml) } \\
\text { Caco2 }\end{array}$ & $\begin{array}{c}\boldsymbol{a} \text { (g protein } / \mathbf{m l}) \\
\text { IEC6 }\end{array}$ \\
\hline 30 & 0.021 & 0.009 \\
60 & 0.026 & 0.013 \\
300 & 0.032 & 0.035 \\
600 & 0.03 & 0.047 \\
\hline
\end{tabular}

\section{DISCUSSION}

We have developed a computational model of glucose transport in the enterocyte that includes the full set of relevant transporters. The model is able to reproduce measurements reported in the literature and can be used to answer physiologically relevant questions about glucose uptake rates and mechanisms. In addition, the capabilities of the CellML framework were exploited to compose existing validated models of individual transporters to create the final model, which provides greater confidence in the implementation and facilitates model reuse and sharing.

\subsection{Comparison With Existing Models}

Our model differs from the Thorsen et al. (2014) model in some important respects.

One of the differences between the two models is in the treatment of sodium and chloride transport at the apical membrane. Thorsen et al. postulate electro neutral one-forone fluxes of these ions to account for the sodium-hydrogen (NHE3) and chloride-bicarbonate (AE1) exchangers and use Goldman-Hodgkin-Katz (GHK) diffusion to model ENaC and CFTR. In contrast, our model takes a more general approach by incorporating the individual transport pathways at the apical membrane (Figure 1). We examined the implications of these modeling choices in Figure 9. Figure 9A shows the ratio of the AE1 flux to NHE3 flux for the simulation conditions of Figure 4. In the Thorsen model this ratio is equal to 1 , whereas the ratio lies in the range 7-8 in our model. Our decision to explicitly model AE1 and NHE3 offers some advantages and testable consequences. First, our model produces the intracellular $\mathrm{pH}$ as an output since $\mathrm{H}+$ concentration is a variable in the model and this provides an additional consistency check. Second, our model can be used to investigate conditions in which the expression/function of AE1 and NHE3 are altered, e.g., impaired absorption in NHE3 knockout mice (Schultheis et al., 1998), reduced chloride absorption and $\mathrm{pH}$ imbalance in $\mathrm{AE} 1$ mutations (Noonan et al., 2005).

Thorsen et al. used sodium and chloride diffusion through both apical and basolateral membrane of the cell. We replaced them with $\mathrm{ENaC}$ and CFTR transporters for sodium and chloride flux in the apical membrane, respectively. Figure 9B shows the ratio of sodium and chloride flux through transporters in our model to the sodium and chloride flux through diffusion in the Thorsen model. It is seen that Chloride flux via CFTR is around 4 times higher than $\mathrm{Cl}^{-}$diffusion and also sodium via $\mathrm{ENaC}$ has around 2 times higher flux compared to $\mathrm{Na}^{+}$ diffusion in Thorsen model. Thus, the contributions of individual transport pathways are significantly different between the models while still providing similar steady state predictions (Figure 4). By incorporating individual transporters our model offers the flexibility to study effects of drugs or diseases that influence the function of these transporters.

\subsection{Parameter Choice and Data Fitting}

Published values from the literature were used for the majority of transport protein kinetic parameters in our model, with a great 

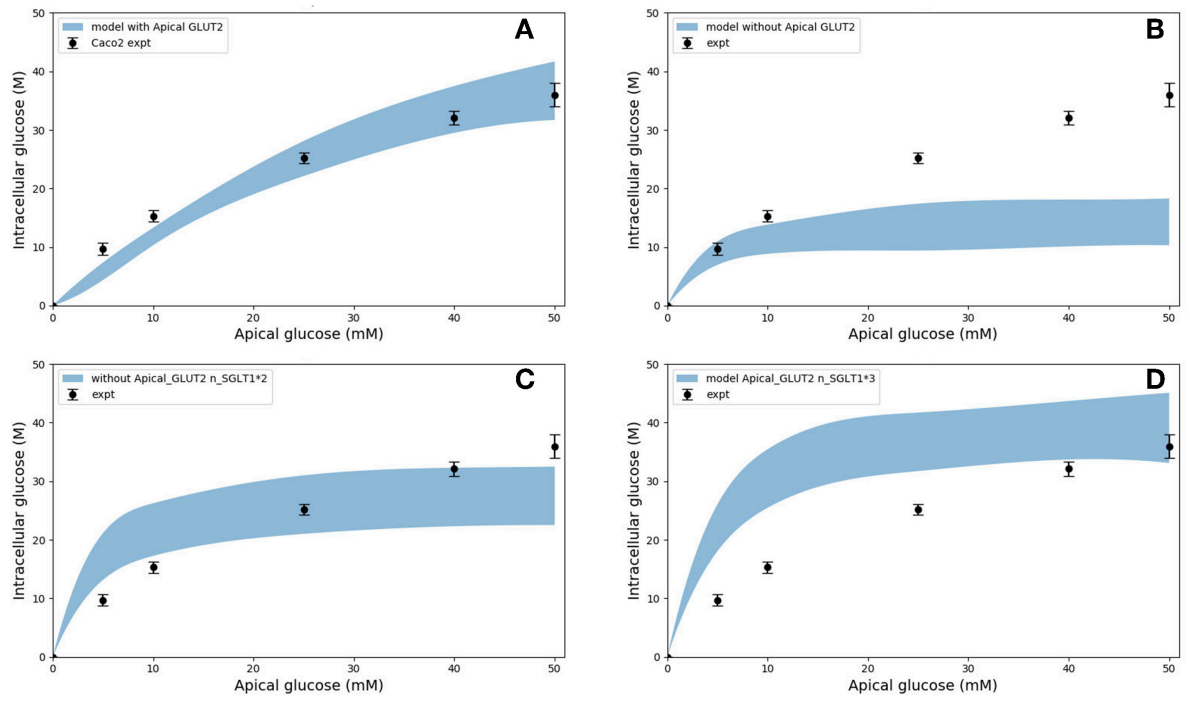

FIGURE 6 | Intracellular glucose concentration vs. extracellular glucose concentration in Caco2 in the presence/absence of Apical GLUT2 with different number of SGLT1 transporter (A) Output of model with apical GLUT2 (B) Model does not have apical GLUT2 (C) model does not have apical GLUT2 and the number of SGLT1 is doubled (D) model does not have apical GLUT2 and the number of SGLT1 is 3-fold higher. Experimental data points and error bars were digitally extracted from Zheng et al. (2012). Strips for the model predictions represent the range of values generated by setting $V_{b}=m V_{c}, m=0.1,1,10,100, \infty$.

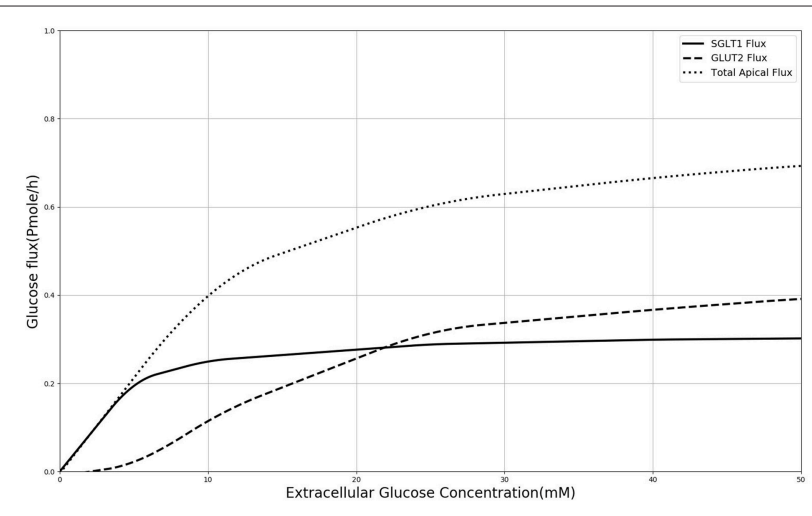

FIGURE 7 | Glucose Flux through SGLT1 and GLUT2 in $600 \mathrm{~s}$ of simulation along with total apical flux of glucose.

deal of information for ohmic models provided by Fong et al. (2016). However, we used a different number of transporters in order to obtain a better fit to the experimental data. These values are shown in the Supplementary Material. The total cellular protein density $(a)$ was used as a fitting parameter to match the model predictions of intracellular glucose with the data of Zheng et al. (2012). For both cell types, the fitted value increased with the duration of glucose exposure (Table 4). Since $c_{\text {pred }}=a c_{\text {expt }}$, this indicates that measured uptake increased at a slower rate with exposure time than predicted by the model. Possible reasons could include desensitization or inactivation of transporters and variations in cell protein density between different experiments. Also, the fitted protein densities are lower than indicative values for the mammalian cells $(0.1-0.2 \mathrm{~g} / \mathrm{ml}, \mathrm{Milo}, 2013)$. The actual

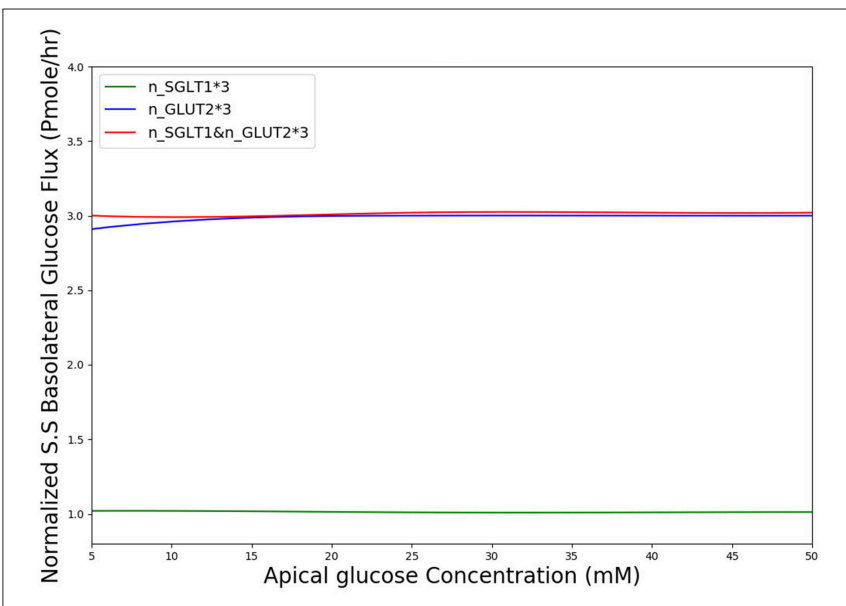

FIGURE 8 | Normalized steady state basolateral glucose flux vs. different stimulus of glucose in the lumen when number of SGLT1 is 3-fold higher(green), number of GLUT2 is 3-fold higher(blue) and number of both SGLT1 \& GLUT2 are 3-fold higher(red).

values of $a$ do not hold much significance since they depend on the cell volume, which were not estimated in the experiments and were assigned arbitrary, realistic values (volume $=1400 \mu \mathrm{m}^{3}$ ) (Buschmann and Manke, 1981; MacLeod et al., 1991; Crowe and Marsh, 1993). Given these caveats, the model produces reasonable fits without the requirement of fine tuning.

It was also necessary to make an assumption about the volume of the basolateral compartment in the comparisons with Zheng et al. (2012) as explained before. Rather than treat this as a fitting parameter, we generated a range of model predictions by 


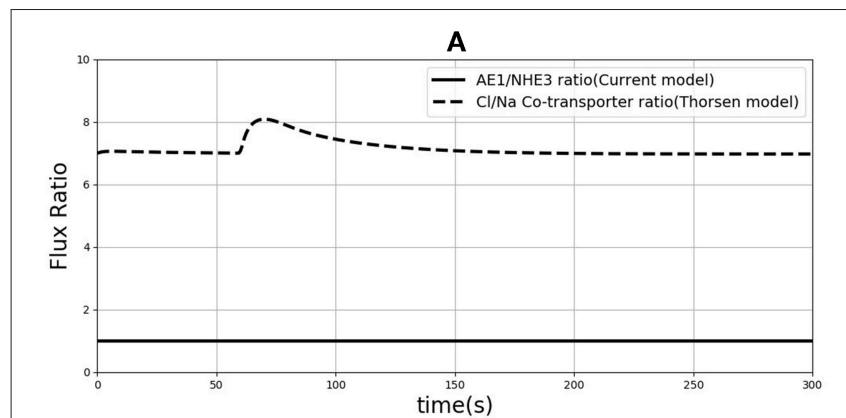

B

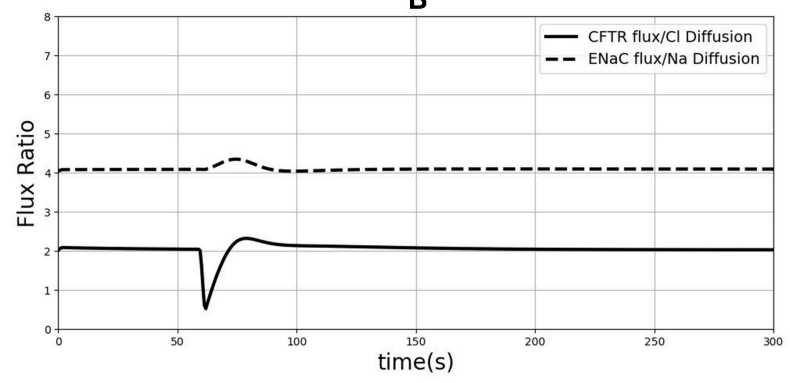

FIGURE 9 | (A) Ratio of sodium flux and Chloride flux through NHE3 and AE1 compare to $\mathrm{NaCl}$ co-transporter flux in the Thorsen model. (B) Chloride flux through CFTR and chloride diffusion ratio compare to the ration of sodium flux through $\mathrm{ENaC}$ and sodium diffusion.

varying the parameter from small ( 0.1 times the cell volume) to large (an infinite compartment) values. The model predictions varied by $<5 \%$ at short exposure durations and about $50 \%$ at long durations (Figure 5) and bracketed the experimental observations in all cases, except few shortest exposures for Caco2. This once again points to the robustness of the model predictions.

\subsection{Role of Apical GLUT2 in Glucose Uptake and Effect of Time}

Figure 10 shows that in both cell lines at short exposure times the glucose uptake has a tendency to be saturated (in 30 and $60 \mathrm{~s}$ ), in longer term (>300 s) Caco2 shows non-saturation glucose uptake (Figure 10A) however IEC6 has a greater tendency to level off even at higher apical glucose concentration (Figure 10B). It has been reported that increasing the glucose concentration in the lumen can cause the apical translocation of GLUT2 (Scow et al., 2011) however, in our model results do not require acute translocation of GLUT2 to the apical membrane. Also Western blots experiments showed higher level of GLUT2 expression in higher extracellular glucose concentration (Kellett and BrotLaroche, 2005); however, in our model density of apical GLUT2 was the same in different concentrations and exposure times. This shows that apical GLUT2 is highly crucial in order to explain the behavior of intracellular glucose absorption.

According to Figure 8, in diabetic patients GLUT2 plays much more important role in the increased glucose absorption compared to SGLT1 regarding the number of transporters. This is in fact a very interesting finding which could be a potential subject of future research into the role of glucose transporter expression levels in diabetic patients.
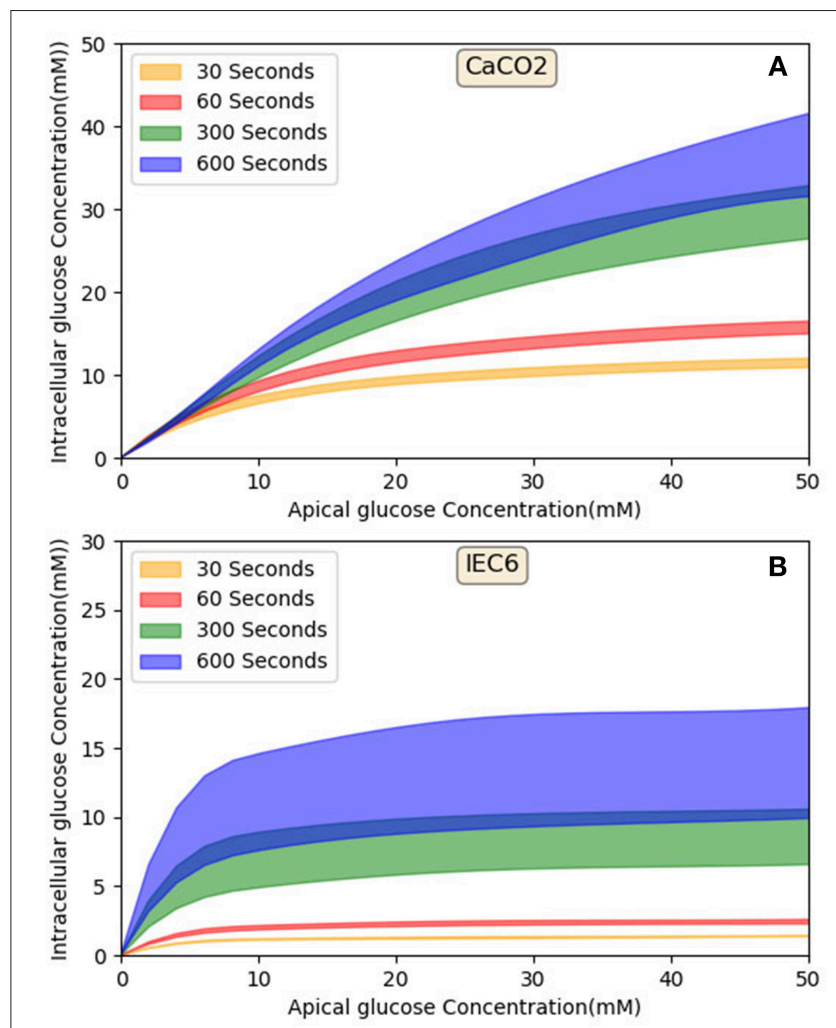

FIGURE 10 | Model output-Intracellular glucose concentration vs. Extracellular glucose concentration in (A) Caco2 cell line and (B) IEC6 cell line.

In summary, we have developed an integrative model of glucose uptake in the enterocyte that incorporates mechanistic descriptions of all relevant transporters and validated it against published measurements and models with minimal parameter tuning. The work utilizes the CellML modeling framework and the Physiome Model Repository to provide a portable, publically available implementation that facilitates sharing, reuse and extension of the model. We expect that the model will provide insight into transport pathways and guide the design and interpretation of experiments to generate and test hypotheses. We have used the model to determine the relative contribution of SGLT1 and GLUT2 to glucose absorption under a range of conditions. We have also evaluated the consequences of altered SGLT1 and GLUT2 expression in diabetes on glucose absorption rates. Potential applications in the future can include predictive modeling of the effect of drugs such as SGLT1 and GLUT2 inhibitors on glucose uptake and ion transport. This model of cellular uptake can be coupled with models of blood flow and metabolism to develop a more complete predictive framework of glucose homeostasis in the body (Nickerson et al., 2015).

\section{AUTHOR CONTRIBUTIONS}

NA, SS, DPN, PJH, and VS contributed conception and design of the study. NA performed the statistical analysis and modeling, wrote the first draft of the manuscript along with sections of the 
manuscript. VS and SS checked the model and validation. All authors contributed to manuscript revision, read and approved the submitted version.

\section{FUNDING}

NA was funded by a doctoral scholarship from the Riddet Centre of Research Excellence, one of ten currently funded by the New

\section{REFERENCES}

Barrett, K. E. and Keely, S. J. (2015). "Electrolyte secretion and absorption in the small intestine and colon," in Yamada's Textbook of Gastroenterology, eds D. K. Podolsky, M. Camilleri, J. G. Fitz, A. N. Kalloo, F. Shanahan, and T. C. Wang (New Jersey, NJ: John Wiley \& Sons, Ltd.), 420-449. doi: 10.1002/9781444303254.ch14

Burant, C. F., Flink, S., DePaoli, A. M., Chen, J., Lee, W. S., Hediger, M. A., et al. (1994). Small intestine hexose transport in experimental diabetes. Increased transporter mrna and protein expression in enterocytes. J. Clin. Invest. 93, 578-585.

Buschmann, R. J. and Manke, D. J. (1981). Morphometric analysis of the membranes and organelles of small intestinal enterocytes. i. fasted hamster. J. Ultra. Res. 76, 1-14. doi: 10.1016/S0022-5320(81)80046-9

Crowe, P. T. and Marsh, M. N. (1993). Morphometric analysis of small intestinal mucosa iv. determining cell volumes. Virchows Archiv. A 422, 459-466. doi: 10.1007/BF01606454

Cuellar, A. A., Lloyd, C. M., Nielsen, P. F., Bullivant, D. P., Nickerson, D. P., and Hunter, P. J. (2003). An overview of cellml 1.1, a biological model description language. Simulation 79, 740-747. doi: 10.1177/0037549703040939

Dyer, J., Garner, A., Wood, I., Sharma, A., Chandranath, I., and Shirazi-Beechey, S. (1997). Changes in the levels of intestinal nat/glucose co-transporter (sglt1) in experimental diabetes. Biochem. Soc. Trans. 25:479S.

Dyer, J., Wood, I., Palejwala, A., Ellis, A., and Shirazi-Beechey, S. (2002). Expression of monosaccharide transporters in intestine of diabetic humans. Am. J. Physiol. Gastroint. Liver Physiol. 282, G241-G248. doi: 10.1152/ajpgi.00310.2001

Fedorak, R. N., Cheeseman, C. I., Thomson, A., and Porter, V. M. (1991). Altered glucose carrier expression: mechanism of intestinal adaptation during streptozocin-induced diabetes in rats. Am. J. Physiol. Gastroint. Liver Physiol. 261, G585-G591. doi: 10.1152/ajpgi.1991.261.4.G585

Fong, S., Chiorini, J. A., Sneyd, J., and Suresh, V. (2016). Computational modeling of epithelial fluid and ion transport in the parotid duct after transfection of human aquaporin-1. Am. J. Physiol. Gastroint. Liver Physiol. 312, G153-G163. doi: 10.1152/ajpgi.00374.2016

Frizzell, R. A., Nellans, H. N., Rose, R. C., Markscheid-Kaspi, L., and Schultz, S. G. (1973). Intracellular cl concentrations and influxes across the brush border of rabbit ileum. Am. J. Physiol. Legacy Content 224, 328-337.

Garny, A. and Hunter, P. J. (2015). Opencor: a modular and interoperable approach to computational biology. Front. Physiol. 6:26. doi: 10.3389/fphys.2015.00026

Gorboulev, V., Schürmann, A., Vallon, V., Kipp, H., Jaschke, A., Klessen, D., et al. (2012). Na+-d-glucose cotransporter sglt1 is pivotal for intestinal glucose absorption and glucose-dependent incretin secretion. Diabetes 61, 187-196. doi: $10.2337 / \mathrm{db} 11-1029$

Hunter, P. J. and Borg, T. K. (2003). Integration from proteins to organs: the physiome project. Nat. Rev. Mol. Cell Biol. 4:237. doi: 10.1038/nrm1054

Ingalls, B. (2012). Mathematical modelling in systems biology: an introduction. Appl. Math. Univ. Waterloo 396, 1-15.

Kellett, G. L. (2012). Comment on: Gorboulev et al. na+-d-glucose cotransporter sglt1 is pivotal for intestinal glucose absorption and glucose-dependent incretin secretion. Diabetes 61, e4-e5. doi: 10.2337/db11-1793

Kellett, G. L. and Brot-Laroche, E. (2005). Apical glut2: a major pathway of intestinal sugar absorption. Diabetes 54, 3056-3062. doi: $10.2337 /$ diabetes.54.10.3056
Zealand Tertiary Education Commission, Funding from RSNZ Marsden (contract UOA1411) is acknowledged.

\section{SUPPLEMENTARY MATERIAL}

The Supplementary Material for this article can be found online at: https:/www.frontiersin.org/articles/10.3389/fphys. 2019.00380/full\#supplementary-material

Kellett, G. L. and Helliwell, P. A. (2000). The diffusive component of intestinal glucose absorption is mediated by the glucose-induced recruitment of glut2 to the brush-border membrane. Biochem. J. 350, 155-162. doi: 10.1042/bj35 00155

Koepsell, H. and Gorboulev, V. (2012). Response to comment on: Gorboulev et al. na+-d-glucose cotransporter sglt1 is pivotal for intestinal glucose absorption and glucose-dependent incretin secretion. Diabetes 61, e5-e5. doi: $10.2337 / \mathrm{db} 12-0061$

König, M., Bulik, S., and Holzhütter, H.-G. (2012). Quantifying the contribution of the liver to glucose homeostasis: a detailed kinetic model of human hepatic glucose metabolism. PLoS Comput. Biol. 8:e1002577. doi: 10.1371/journal.pcbi.1002577

MacLeod, R. J., Hamilton, J., Bateman, A., Belcourt, D., Hu, J., Bennett, H., and Solomon, S. (1991). Corticostatic peptides cause nifedipine-sensitive volume reduction in jejunal villus enterocytes. Proc. Natl. Acad. Sci. U.S.A. 88, 552-556. doi: $10.1073 /$ pnas.88.2.552

Milo, R. (2013). What is the total number of protein molecules per cell volume? a call to rethink some published values. Bioessays 35, 1050-1055 doi: 10.1002/bies.201300066

Nellans, H. N., Frizzell, R. A., and Schultz, S. G. (1973). Coupled sodium-chloride influx across the brush border of rabbit ileum. Am. J. Physiol. Legacy Content $225,467-475$.

Nellans, H. N., and Schultz, S. G. (1976). Relations among transepithelial sodium transport, potassium exchange, and cell volume in rabbit ileum. J. Gen. Physiol. 68, 441-463.

Nickerson, D. P., Ladd, D., Hussan, J. R., Safaei, S., Suresh, V., Hunter, P. J., et al. (2015). Using cellml with opencmiss to simulate multi-scale physiology. Front. Bioeng. Biotechnol. 2:79. doi: 10.3389/fbioe.2014.00079

Noonan, W. T., Woo, A. L., Nieman, M. L., Prasad, V., Schultheis, P. J., Shull, G. E., et al. (2005). Blood pressure maintenance in nhe3-deficient mice withtransgenic expression of nhe3 in small intestine. Am. J. Physiol. Regul. Integrat. Compar. Physiol. 288, R685-R691. doi: 10.1152/ajpregu. 00209.2004

Okada, Y., Irimajiri, A., and Inouye, A. (1976). Intracellular ion concentrations of epithelial cells in rat small intestine effects of external potassium ions and uphill transports of glucose and glycine. Jap. J. Physiol. 26, 427-440.

Østby, I., Øyehaug, L., Einevoll, G. T., Nagelhus, E. A., Plahte, E., Zeuthen, T., et al. (2009). Astrocytic mechanisms explaining neural-activityinduced shrinkage of extraneuronal space. PLoS Comput Biol. 5:e1000272. doi: 10.1371/journal.pcbi.1000272

Palk, L., Sneyd, J., Shuttleworth, T. J., Yule, D. I., and Crampin, E. J. (2010). A dynamic model of saliva secretion. J. Theor. Biol. 266, 625-640. doi: 10.1016/j.jtbi.2010.06.027

Palumbo, P., Ditlevsen, S., Bertuzzi, A., and De Gaetano, A. (2013). Mathematical modeling of the glucose-insulin system: a review. Math. Biosci. 244, 69-81. doi: 10.1016/j.mbs.2013.05.006

Parent, L., Supplisson, S., Loo, D. D., and Wright, E. M. (1992). Electrogenic properties of the cloned na+/glucose cotransporter: Ii. a transport model under nonrapid equilibrium conditions. J. Membr. Biol. 125, 63-79.

Pradhan, R. K., Vinnakota, K. C., Beard, D. A., and Dash, R. K. (2013). Chapter 5 : Carrier-mediated Transport Through Biomembranes (Elsevier).

Riz, M., and Pedersen, M. G. (2015). Mathematical modeling of interacting glucose-sensing mechanisms and electrical activity underlying glucagon-like peptide 1 secretion. PLoS Comput. Biol. 11:e1004600. doi: 10.1371/journal.pcbi.1004600 
Röder, P. V., Geillinger, K. E., Zietek, T. S., Thorens, B., Koepsell, H., and Daniel, H. (2014). The role of sglt1 and glut2 in intestinal glucose transport and sensing. PLoS ONE 9:e89977. doi: 10.1371/journal.pone.0089977

Rose, R. C., and Schultz, S. G. (1971). Studies on the electrical potential profile across rabbit ileum. J. Gen. Physiol. 57, 639-663. doi: 10.1085/jgp.57.6.639

Schedl, H. P., and Wilson, H. D. (1971). Effects of diabetes on intestinal growth in the rat. J. Exp. Zool. 176, 487-495. doi: 10.1002/jez.1401760410

Schultheis, P. J., Clarke, L. L., Meneton, P., Miller, M. L., Soleimani, M., Gawenis, L. R., et al. (1998). Renal and intestinal absorptive defects in mice lacking the nhe3 nat/h+ exchanger. Nat. Genet. 19:282. doi: 10.1038/969

Scow, J. S., Tavakkolizadeh, A., Zheng, Y., and Sarr, M. G. (2011). Acute "adaptation" by the small intestinal enterocyte: a posttranscriptional mechanism involving apical translocation of nutrient transporters. Surgery 149, 601-605. doi: 10.1016/j.surg.2011.02.001

Shimada, T., and Hoshi, T. (1987). Role of nat/h+ antiport in intracellular ph regulation by rabbit enterocytes. Biochim. Biophys. Acta 901, 265-272. doi: 10.1016/0005-2736(87)90123-4

Thorsen, K., Drengstig, T., and Ruoff, P. (2014). Transepithelial glucose transport and nat/k+ homeostasis in enterocytes: an integrative model. Am. J. Physiol. Cell Physiol. 307, C320-C337. doi: 10.1152/ajpcell.00068.2013

Vogalis, F. (2000). Potassium channels in gastrointestinal smooth muscle. J. Auton. Pharmacol. 20, 207-219.

Weinstein, A. M. (1995). A kinetically defined $n a+/ h+$ antiporter within a mathematical model of the rat proximal tubule. J. Gen. Physiol. 105, 617-641.
Weinstein, A. M. (2000). A mathematical model of the outer medullary collecting duct of the rat. Am. J. Physiol. Renal Physiol. 279, F24-F45. doi: 10.1152/ajprenal.2000.279.1.F24

Weinstein, A. M. (2015). A mathematical model of the rat nephron: glucose transport. Am. J. Physiol. Renal Physiol. 308, F1098-F1118. doi: 10.1152/ajprenal.00505.2014

Yu, T., Lloyd, C. M., Nickerson, D. P., Cooling, M. T., Miller, A. K., Garny, A., et al. (2011). The physiome model repository 2. Bioinformatics 27, 743-744. doi: 10.1093/bioinformatics/btq723

Zheng, Y., Scow, J. S., Duenes, J. A., and Sarr, M. G. (2012). Mechanisms of glucose uptake in intestinal cell lines: role of glut2. Surgery 151, 13-25. doi: 10.1016/j.surg.2011.07.010

Conflict of Interest Statement: The authors declare that the research was conducted in the absence of any commercial or financial relationships that could be construed as a potential conflict of interest.

Copyright (๑ 2019 Afshar, Safaei, Nickerson, Hunter and Suresh. This is an openaccess article distributed under the terms of the Creative Commons Attribution License (CC BY). The use, distribution or reproduction in other forums is permitted, provided the original author(s) and the copyright owner $(s)$ are credited and that the original publication in this journal is cited, in accordance with accepted academic practice. No use, distribution or reproduction is permitted which does not comply with these terms. 